\title{
LA SALUD HOLISTICA EN LOS CUIDADOS APLICADOS POR ESTUDIANTES DE LA FAEN/UNJBG
}

Responsable: Enf. Yolanda Paulina Torres Chávez Miembros: Mgr Eloina Inés Tejada Monroy Mgr. Ingrid Maria Manrique Tejada

\section{RESUMEN}

El presente trabajo tuvo como resultado que una gran mayoría de estudiantes de la FAEN, conocen el significado que tiene el cuidado en la profesión de enfermería, y que en el entorno inmediato donde se ejerce la profesión, existe un conjunto de normas que determinan que el acto de cuidar no está centrado en la persona desde una visión de salud holística. Por otro lado, también una gran mayoría de estudiantes consideran que la salud holística y humanística del cuidado es el eje central de la formación profesional. Finalmente, se concuerda con los estudios realizados por José Bermejo (1999), que revelan que las personas valoran más los aspectos del cuidado relacionados con la comunicación, el afecto y la atención que presta la enfermera.

\section{INTRODUCCION}

La evolución del hombre a través de su historia es muy compleja y más aún en el cuidado de su salud.

El cuidado, desde un enfoque de salud holística, considera: "A las personas en su dimensiones física, intelectual, social, emocional y espiritual, es decir, como un todo integral", coincidiendo con las definiciones que Bermejo (1999) hace en relación con la salud holística.

La importancia del presente estudio radica en que tanto la docente como la estudiante profundicen el conocimiento y la praxis sobre salud holística para suministrar cuidados humanizados, basados en la calidad y calidez de persona que cuidan a personas. Y concluimos que tanto docentes como estudiantes administren esos cuidados con el enfoque de la salud holística.

\section{MARCOTEORICO}

Para el desarrollo del marco teórico las investigadoras hemos considerado los siguientes temas:

1. Salud Holística: Significa considerar a la persona en toda sus dimensiones(físico, intelectual, social emocional, espiritual).
2. Estilo de Acompañamiento Holístico: Es el que genera la salud holística y esta seria, la experiencia de la persona de armonia y responsabilidad en la gestión de la propia vida, de los propios recursos, de sus límites y disfunciones en cada una de sus dimensiones.

3. El Cuidado: Es la capacidad para hacer algo o tener la habilidad para hacer algo, para desarrollar una determinada tarea por tanto el ejercicio del cuidado se relaciona con la tarea de capacitar por que cuando uno cuida a alguien de verdad, lo que trata de conseguir es que ese sujeto pueda ganar niveles de autonomía, sin necesidad de recurrir a otro.

4. Constructor éticos del cuidar: Son virtudes básicos e ineludibles que se requieren para cuidar al ser humano en sufrimiento físicomental, social y espiritual. Segun Goria Brykznska, los puntos de la tarea de cuidar puede sintetizarse en los siguientes constructor éticos morales:

- Compasión

- Competencia

- Confianza

- Conciencia

- Confidencialidad

Los constructores no se refieren al ser del cuidar, es decir, al cuidar tal y como se desarrolla en el mundo de la vida, si no que se refieren a su "deber ser". 
5. Elementos para el Cuidado: Entre los rasgos categoriales del cuidar, se puede enumerar los siguientes elementos:

- El Tacto

- Capacidad de escuchar

- El sentido del humor

\section{III.OBJETIVOS:}

Objetivo General: Determinar la aplicación de la salud holistica en los cuidados brindados por los estudiantes de la Facultad de Enfermeria (FAEN) de la Universidad Nacional Jorge Basadre Grohmann(UNJBG).

\section{Objetivos Específicos:}

1. Determinar el conocimiento sobre salud holistica que tienen los estudiantes de la Facultad de Enfermeria de la UNJBG.

2. Determinar la aplicación del cuidado de enfermería con enfoque en salud holistica por parte de los estudiantes de la Facultad de Enfermería de la UNJBG.

\section{MATERIALES Y MÉTODOS}

1. Tipo de Investigación: La presente investigación se categoriza como investigación básica.

2. Diseño de la Investigación: Por la naturaleza del estudio, en el proceso de investigación se aplicará el diseño: investigación descriptiva y correlacional.

\section{Población y Muestra de Estudio:}

Población de Estudio: La población o universo del estudio está constituido por los estudiantes de 3ero, 4to y 5to años de estudios haciendo un total 100 estudiantes.

\section{RESULTADO}

Cuadro 01: Conocimiento de Conceptos de Salud, Salud Holistica y Cuidado de los Estudiantes de la Facultad de Enfermería, UNJBG -2007.

\begin{tabular}{|l|c|c|c|c|c|c|c|c|}
\hline \multirow{2}{*}{ CONOCIMIENTO } & \multicolumn{2}{|c|}{ Siempre } & \multicolumn{2}{|c|}{ A Veces } & \multicolumn{2}{|c|}{ Nunca } & \multicolumn{2}{|c|}{ Total } \\
\cline { 2 - 9 } & $\mathrm{N}^{0}$ & $\%$ & $\mathrm{~N}^{\circ}$ & $\%$ & $\mathrm{~N}^{\circ}$ & $\%$ & $\mathbf{N}^{\circ}$ & $\%$ \\
\hline Salud & 100 & 100 & 00 & 00 & 00 & 00 & 100 & 100 \\
\hline Salud Holistica & 60 & 60 & 32 & 32 & 08 & 08 & 100 & 100 \\
\hline Cuidado & 100 & 100 & 00 & 00 & 00 & 00 & 100 & 100 \\
\hline
\end{tabular}

Fuente: Cuestionario aplicado a estudiantes de la FAEN-UNJBG

\section{Interpretación}

El Cuadro 1 muestra que en su totalidad conocen el término salud y cuidado pero sólo en su mayoría conocen el concepto de salud holistica.

Lo que nos permite aseverar que la mayoría de los estudiantes tienen el conocimiento de salud holistica, habiéndolo adquirido en su formación preprofesional universitaria.

Cuadro 02: Relaciona los Conceptos de Salud, Salud Holística y Cuidado por parte de los Estudiantes de Facultad de Enfermeria UNJBG - 2007.

\begin{tabular}{|l|c|c|c|c|c|c|c|c|}
\hline \multirow{2}{*}{ RELACIONA } & \multicolumn{2}{|c|}{ Siempre } & \multicolumn{2}{l|}{ A Veces } & \multicolumn{2}{l|}{ Nunca } & \multicolumn{2}{l|}{ Total } \\
\cline { 2 - 9 } & $\mathbf{N}^{0}$ & $\%$ & $\mathbf{N}^{\circ}$ & $\%$ & $\mathbf{N}^{\circ}$ & $\%$ & $\mathbf{N}^{0}$ & $\%$ \\
\hline Salud y cuidado & 100 & 100 & 00 & 00 & 00 & 00 & 100 & 100 \\
\hline Salud Holistica y cuidado & 65 & 65 & 26 & 26 & 09 & 9 & 100 & 100 \\
\hline
\end{tabular}

Fuente: Cuestionario aplicado a estudiantes de la FAEN-UNJBG

\section{Interpretación}

El Cuadro 2 muestra que en su totalidad relacionan el término salud con cuidado y la mayoría relacionan salud holística y cuidado.

Lo que nos permite afirmar que la mayoría de los estudiantes conocen la importancia de la relación de salud holistica y cuidado para la persona.

Cuadro 03: Aplicación de la Salud Holística por parte del Profesional y el Estudiante de Enfermeria, según la Percepcion de los Estudiantes de la Facultad de Enfermeria UNJBG 2007.

\begin{tabular}{|l|c|c|c|c|c|c|c|c|}
\hline \multirow{2}{*}{ APLICACIÓN } & \multicolumn{2}{|c|}{ Siempre } & \multicolumn{2}{|c|}{ A Veces } & \multicolumn{2}{|c|}{ Nunca } & \multicolumn{2}{|c|}{ Total } \\
\cline { 2 - 9 } & $\mathbf{N}^{0}$ & $\%$ & $\mathbf{N}^{\circ}$ & $\%$ & $\mathbf{N}^{0}$ & $\%$ & $\mathbf{N}^{\circ}$ & $\%$ \\
\hline La Profesional de Enfermeria & 10 & 10 & 60 & 60 & 30 & 30 & 100 & 100 \\
\hline El Estudiante de Enfermeria & 30 & 30 & 65 & 65 & 05 & 5 & 100 & 100 \\
\hline
\end{tabular}

Fuente: Cuestionario aplicado a estudiantes de la FAEN-UNJBG.

\section{Interpretación}

El Cuadro 03 muestra que la mayoría de las profesionales de enfermería, según la percepción de la estudiante de enfermería, aplican a veces la salud holística en sus cuidados; de igual manera la gran mayoría de los estudiantes aplican la salud holistica a veces.

Lo que nos permite concluir que tanto profesionales como estudiantes en su gran mayoria aplican la salud holistica en los cuidados que prestan.

Cuadro 04: Durante la Formación Profesional se le enseñó sobre Salud, Salud Holistica y 
Cuidado en la Facultad de Enfermería UNJBG 2007.

\begin{tabular}{|l|r|r|r|r|r|r|r|r|}
\hline \multirow{2}{*}{ ENSEÑO } & \multicolumn{2}{|c|}{ Siempre } & \multicolumn{2}{|c|}{ A Veces } & \multicolumn{2}{|c|}{ Nunca } & \multicolumn{2}{c|}{ Total } \\
\cline { 2 - 9 } & $N^{0}$ & \multicolumn{1}{|c|}{$\%$} & $N^{0}$ & $\%$ & $N^{0}$ & $\%$ & $N^{0}$ & $\%$ \\
\hline Salud & 100 & 100 & 00 & 00 & 00 & 00 & 100 & 100 \\
\hline Salud Holistica & 45 & 45 & 49 & 49 & 06 & 6 & 100 & 100 \\
\hline Cuidado & 100 & 100 & 00 & 00 & 00 & 00 & 100 & 100 \\
\hline
\end{tabular}

Fuente: Cuestionario aplicado a estudiantes de la FAEN-UNJBG

\section{Interpretación}

El Cuadro 4 muestra que a la totalidad de los estudiantes se les enseñó sobre salud y cuidado; y la mitad de estudiantes refieren que se les enseño el concepto de salud holística a veces.

Ello nos permite afirmar que en la mayoría de los estudiantes falta profundizar el conocimiento de salud holistica.

Cuadro 05: Momento de la Aplicación del Cuidado por parte de los Estudiantes de la Facultad de Enfermería - UNJBG - 2007.

\begin{tabular}{|l|r|r|r|r|r|c|c|c|}
\hline \multirow{2}{*}{ CUIDADO } & \multicolumn{2}{|c|}{ Siempre } & \multicolumn{2}{|c|}{ A Veces } & \multicolumn{2}{|c|}{ Nunca } & \multicolumn{3}{|c|}{ Total } \\
\cline { 2 - 8 } & $\mathbf{N}^{0}$ & $\%$ & $\mathbf{N}^{0}$ & $\%$ & $\mathbf{N}^{\circ}$ & $\%$ & $\mathbf{N}^{0}$ & $\%$ \\
\hline Fisico & 100 & 100 & 00 & 00 & 00 & 00 & 100 & 100 \\
\hline Mental & 32 & 32 & 47 & 47 & 21 & 21 & 100 & 100 \\
\hline Social & 31 & 31 & 51 & 51 & 18 & 18 & 100 & 100 \\
Espiritual & 05 & 5 & 32 & 32 & 63 & 63 & 100 & 100 \\
Emocional & 50 & 50 & 50 & 50 & 00 & 00 & 100 & 100 \\
\hline
\end{tabular}

Fuente: Cuestionario aplicado a estudiantes de la FAEN-UNJBG.

\section{Interpretación}

El Cuadro 5 muestra que la totalidad de estudiantes aplican sus cuidados en la dimensión física; y en la mayoría nunca aplican la dimensión espiritual.

Lo que nos permite sostener que los estudiantes dirigen más su cuidado a la dimensión física, dejando de lado las otras áreas indispensables para el cuidado desde un enfoque de salud holística

\section{VI.CONCLUSIONES}

1.Los estudiantes tienen conocimiento en su totalidad sobre cuidado y salud, recibido en su formación profesional, pero hay que reforzar sobre salud holistica para que sea un "cuidado más humanizado".

2.Se observa que es el entorno inmediato donde la estudiante y la profesional de enfermería desarrollan su trabajo, tienen sistemas complejos, con pautas y normas establecidas, lo cual de alguna forma está determinando que el acto de cuidar no esté centrado en la persona desde una concepción de salud holistica.

3.Considerar la visión holística y humanista del cuidado en nuestra disciplina como eje central en la formación de las estudiantes de enfermeria, incorporando teorías de la comunicación transpersonal consideradas claves en el cuidado.

4.Es difícil mantener los valores humanitarios en el acto de cuidar en las instituciones públicas de salud en donde parecen invisibilizarse los cuidados de enfermeria por las labores biomédicas, pero los estudiantes deben seguir aplicando la salud holística y poder cambiar y mejorar la forma del cuidado hacia la persona, la familia y la comunidad.

\section{RECOMENDACIONES}

Es necesario que a partir de los datos presentados se desarrollen nuevas investigaciones que permitan determinar otros factores que influyen en la profundizacion del conocimiento de salud holistica.

Los docentes responsables de cada curso deberan de fortalecer mas sobre salud holistica y poder brindar un mejor cuidado humanizado.

\section{BIBLIOGRAFÍA}

Álvarez, F. "La experiencia humana de la salud desde una óptica cristiana", en Labor Hospitalaria, 219(1991), p. 33.

Garcia Roca, J. Contra la exclusión. Responsabilidad politica e iniciativa social, Santander, Sal Terrae, 1995, pp. 9-15.

Bermejo, J.C. Relación de ayuda y marginación, in Camillianum, Roma, 1999 (20), pp. 279-296.

Laspina, M. (1996). "Concepto de espiritualidad en el contexto de la enfermeria". Boletín Impulso, 6, 27-29.

Potter, P. \& Perry, A. (1997). Fundamentos de enfermeria, conceptos, proceso y práctica (4ed.). St. Louis: Mosby. 


\section{ANEXO}

\section{ENCUESTA}

Año de Estudios:

Fecha:

Edad:

\begin{tabular}{|c|c|c|c|}
\hline & Siempre & A veces & Nunca \\
\hline \multicolumn{4}{|l|}{ Conoces sobre salud. } \\
\hline \multicolumn{4}{|l|}{ Conoces sobre salud holística. } \\
\hline \multicolumn{4}{|l|}{$\begin{array}{l}\text { Salud Holistica es un completo estado de bienestan } \\
\text { físico, mental y social y no meramente la ausencia de } \\
\text { enfermedad o incapacidad. }\end{array}$} \\
\hline \multicolumn{4}{|l|}{ Conoce lo que es cuidado. } \\
\hline \multicolumn{4}{|l|}{$\begin{array}{l}\text { El cuidado humano con una visión holistica conlleva } \\
\text { actuar en forma eficaz y comprometida, con la práctica de } \\
\text { valores, en busca de la ciudadania garantizando un } \\
\text { bienestar físico,mental, social y espiritual de la persona } \\
\text { individual, la familia y la comunidad. }\end{array}$} \\
\hline \multicolumn{4}{|l|}{$\begin{array}{l}\text { Cualidades éticas que debe tener una enfermera (o) son. } \\
\text { Compasión, Competencia, Confianza, Confidencialidad } \\
\text { Conciencia. }\end{array}$} \\
\hline \multicolumn{4}{|l|}{ Se relaciona cuidado y salud. } \\
\hline \multicolumn{4}{|l|}{ Se relaciona cuidados y salud holística. } \\
\hline \multicolumn{4}{|l|}{ La enfermería aplica la salud holistica en sus cuidados. } \\
\hline \multicolumn{4}{|l|}{$\begin{array}{l}\text { Durante su formación profesional se les enseño sobre } \\
\text { salud holistica. }\end{array}$} \\
\hline \multicolumn{4}{|l|}{$\begin{array}{l}\text { Las dimensiones de la salud holistica son físicas, mentales } \\
\text { o intelectuales, sociales, emocionales y espirituales } \\
\text { religiosas. }\end{array}$} \\
\hline \multicolumn{4}{|l|}{$\begin{array}{l}\text { Los estudiantes de enfermería aplican la salud holistica en } \\
\text { sus cuidados. }\end{array}$} \\
\hline \multicolumn{4}{|l|}{\begin{tabular}{|l|l|} 
Durante su formación se les enseñó sobre cuidados. \\
\end{tabular}} \\
\hline \multicolumn{4}{|l|}{ Piensa que hay diferencia entre salud y salud holística. } \\
\hline \multicolumn{4}{|l|}{$\begin{array}{l}\text { Se brinda un cuidado adecuado: teniendo tacto con la } \\
\text { persona, escuchamos atentamente y teniendo sentido de } \\
\text { humor. }\end{array}$} \\
\hline \multicolumn{4}{|l|}{ El cuidado implica : cuidar de la persona sana o enferma. } \\
\hline \multicolumn{4}{|l|}{ Cuida la parte fisica de la persona que está cuidando. } \\
\hline \multicolumn{4}{|l|}{$\begin{array}{l}\text { Cuida la parte intelectual de la persona que está } \\
\text { cuidando. }\end{array}$} \\
\hline \multicolumn{4}{|l|}{ Cuida la parte social de la persona que está cuidando. } \\
\hline $\begin{array}{l}\text { Cuida la parte emocional de la persona que está } \\
\text { cuidando. }\end{array}$ & & & \\
\hline
\end{tabular}

